PRINT ISSN 1119-8362

ELECTRONIC ISSN 1119-8362
Full-text Available Online at

J. Appl. Sci. Environ. Manage. https://www.ajol.info/index.php/jasem Vol. 23 (3) 417-423 March 2019 http://ww.bioline.org.br/ja

\title{
Performance Appraisal of the Traffic Intersection Complex at Mokola, Ibadan, Nigeria
}

\section{OYEDEPO, OJ; *OGUNWOLU, L; AKINFALA, L}

\author{
${ }^{1}$ Department of Civil and Environmental Engineering, Federal University of Technology, Akure, Nigeria \\ ${ }^{2}$ Department of Systems Engineering, Faculty of Engineering, University of Lagos, Nigeria \\ ${ }^{3}$ Department of Geography, Faculty of Social Sciences, University of Lagos, Nigeria \\ *Corresponding Author Email: fogunwolu@unilag.edu.ng; Other Authors Email: oyedepoo@yahoo.co.uk; lanrewaju.akinfala@gmail.com
}

\begin{abstract}
Throughput time reduction is an integral part of transportation benefit analysis and traffic engineering optimization objectives. It is for this reason, that a large majority of transportation projects view throughput time reduction as one of their principal objectives. An appraisal of the performance of a traffic intersection complex at Mokola, Ibadan, Nigeria using a conflict intensity approach is presented. This work was carried out in order to quantify the effects of the flyover on the reduction of throughput times of vehicular traffic at the intersection. Conflict intensities were measured through traffic volume counts at various conflict points (through, merging and diverging locations). A floating vehicle equipped with a GPS was adopted to capture throughput times through several trial runs at the intersection. Statistical relationships were established between conflict intensities and throughput times using regression analysis. These relationships served as a framework for simulation for generating travel times (augmented), assuming the absence of a flyover. The results show a statistically significant reduction in throughput times due to the presence of the flyover with p-value of $<0.0001$ at alpha level of 0.05 using Wilcoxon-Signed rank test. Specifically, reductions of 40.2 seconds (33\%) for northbound traffic and 27.6 seconds $(28.8 \%)$ for southbound traffic were observed. This work has shown that the flyover reduced throughput times. The construction of the flyover is therefore justified and its residual potentials are enormous to handle traffic growth in the area in the near future.
\end{abstract}

DOI: https://dx.doi.org/10.4314/jasem.v23i3.8

Copyright: Copyright () 2019 Oyedepo et al. This is an open access article distributed under the Creative Commons Attribution License (CCL), which permits unrestricted use, distribution, and reproduction in any medium, provided the original work is properly cited.

Dates: Received: 14 September 2018; Revised: 19 February 2019; Accepted 22 March 2019

Keywords: Intersection Performance, Conflict Intensities, Flyover, Throughput times

The traffic intersection is one of such transportation/traffic infrastructures that pose challenges of lowered level of service occasioned by congestion and traffic sprawl especially in the urban centers. They are critical points within urban networks (MUTCD, 2009), consequently, traffic operation problems at intersections can ripple through the entire transport network of a locality. Intersections are locations where complex and heavy maneuverings occur; thus, they must be properly designed and equipped to handle traffic for easy and safe turnings. Intersections typically have lower per lane capacity in comparison to other roadway facilities (Alejandro and Mark 2003) and hence, they typically experience diminished performance levels in comparison to facilities such as freeways and highways which have fewer interruptions in flow of traffic because access to them is very limited. Furthermore, intersections accommodate many other non-motorized and conflicting modes of travel such as pedestrians and bicycles. This introduces more challenges to the operations of the intersection. The intersection is thus a point of decision on the roadway by both drivers and pedestrians vying for space. Furthermore, the way intersections are designed essentially leads to conflicts. Thompson et al 2009, highlighted four main types of conflict at intersections namely: sequential, merging, diverging and crossing conflicts. The issue of conflict poses an even more serious problem in developing countries, largely because of nonadherence to lanes and our vehicular mix. It is also common knowledge in traffic engineering that a typical cross intersection has 32 potential conflict points. It is for this reason that various types of controls such as STOP signs, GIVE WAY, and traffic signals are installed. These controls are primarily designed to assign right-of-way efficiently and safely to conflicting movements (FHWA, 2008). This informs the reason why when an intersection is not well designed, controlled or managed; it could be an incessant location for accidents and excessive delays and queues. When traffic volume is light to moderate and these controls are installed appropriately, they often prove effective. However, some situations arise when traffic volume is very high and traffic signals or other forms of controls lead to excessive delay and queues. In such situations, grade separation (flyovers) becomes paramount. Flyovers are constructed when other measures such as signal timing optimization, lane additions/expansions may fail to yield desired 
performance results. Flyovers increase capacity and reduce delays and queues. There is also increased speeds and reduced collisions because of reduced interactions (Texas A\&M Transport Institute, 2018). This paper focuses on the effect of traffic volume at conflict points within a flyover intersection; with the aim of assessing the effect of the flyover on performance as well as through travel time prediction and comparison. The primary purpose of computing a performance measure is to assess or quantify the impact of a project's traffic operations objective (FHWA, 2007). Travel time has been used extensively as a performance measure at intersections in a wide variety of studies (Day et al 2012, Remias et al 2013; Davis and Xiong, 2007). Our present study also adopts travel time in order to assess the efficacy of grade separation at the intersection of interest. More specifically, throughput time was adopted as the performance measure because it incorporates delay experienced by vehicles as well as free flow time.

\section{MATERIALS AND METHODS}

The appraisal of the intersection was based on existing traffic flow data at-grade and the projected traffic flow data in the absence of grade-separation (the flyover) taken as the factored addition of current traffic on flyover and at grade. For each of these, turning movement counts were taken from which the flow intensities at each potential conflict points were computed together with corresponding travel times or throughput across the intersection in the direction of the flyover. Traffic turning movements were counted for all traffic movements at-grade while travel times or thoroughfare times were measured from the at-grade entrance of the flyover to its exit point for both southbound and northbound flow directions for three periods of the day of two hours each in the morning peak, afternoon and evening peak flow periods.

Three major types of vehicular conflicts are identifiable on the Mokola intersection. These are: Merging conflict $(M)$ : This is a conflict scenario in which two or more vehicle streams coming from different directions vie to turn to the same direction. The more the number of contributing approaches to the merging point the more severe the conflict magnitude and the challenge of its resolution.

Cross-conflict $(C)$ : A conflict scenario in which two vehicles travelling in opposite directions most times perpendicular meet at a point. This type of conflict is the most severe and most difficult to resolve. Collision caused by this type of conflict is also the most severe of all.

Diverging Conflict (D): This is a conflict scenario in which vehicles coming from the same direction veer at the point to take different directions. Except for possible delay due to the maneuvering and possible merging conflict ahead of the leading vehicles, this type of conflict does not cause much delay as the other two types. For simplicity, they are like conflict resolution or negative conflicts points.

Conflict Configurations at Mokola Intersection: The vehicle traffic conflict configuration at Mokola flyover in Ibadan as it currently exists after the construction of the flyover combines all the above defined conflict types. It should be noted that the primary aim of the flyover is to resolve or significantly reduce the traffic conflicts before its construction. Hence, a measure of the comparative effectiveness of the construction of the flyover after construction and the situation if it had not been constructed is a measure of the desirability, effectiveness and efficiency of the flyover. The figure below is the complete configuration of the traffic conflicts on the corridor as currently being used. It should be noted that all approaching roadways to the intersection hold two lanes of traffic each but commercial activities have literarily taken over one lane each on each approach roadway. Hence, it is only practicable to idealize the approach roadways as carrying one lane of traffic.

If this assumption is contrary to the ideal of construction of a flyover at Mokola, it will first and foremost be an indictment on poor corridor management before it can squarely be laid to inefficiency of the flyover. Even when the constructed flyover is found to be efficient and desirable as constructed, it can be argued that with better intersection traffic corridor management, the efficiency could be better enhanced.

Method of Quantification of Conflict Intensities: At grade and parallel to the direction of travel of the separated traffic on the flyover, there are eight conflict points in each direction that the separated traffic would have passed through (see Figure 1 below). These are made of 3 merging, 2 cross and 3 diverging conflicts in each direction of travel along the flyover layout. Traffic counts were made at the intersection at locations A, B, C, D, E, F and G as shown in Figure 1. Corresponding turning movements of traffic monitored and counted at each position are labeled in identical lower-case letters. For example, at Location A, turning movement's a1, a2 and a3 were monitored and counted. From these counts, the traffic intensities at the different conflict points were computed for both directions of the flyover (Southbound and Northbound) as in figure 1. 


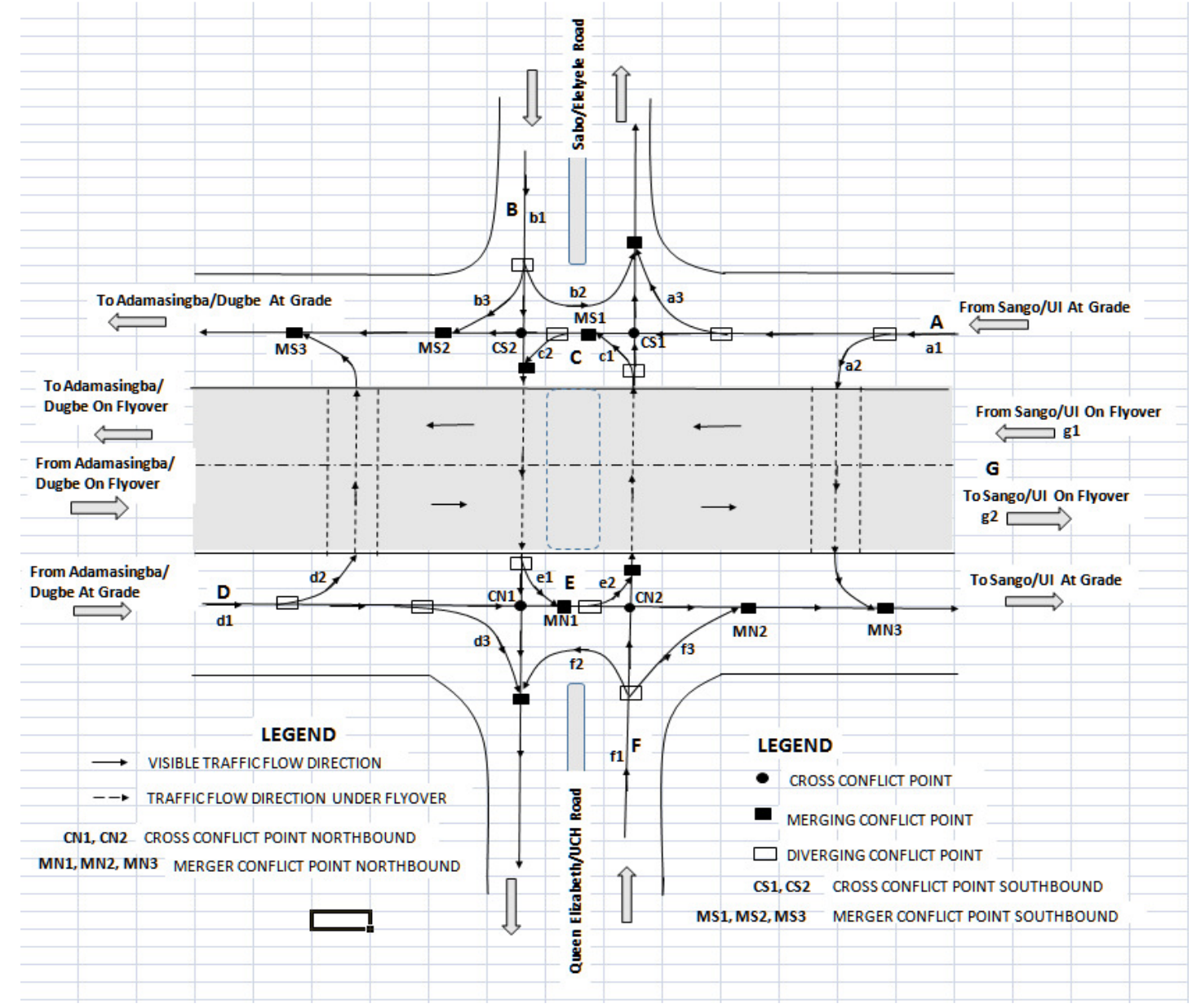

Fig 1: Vehicular Traffic Conflict Configuration at Mokola Flyover Intersection Corridor

Note that the Diverging conflicts were neglected in computation as they contribute almost insignificant time to delay and hence throughput times.

Southbound Flow (UI/Sango to Dugbe/Adamasingba direction)

$$
\begin{aligned}
& \mathrm{C}_{\mathrm{S}} 1=\mathrm{a} 1-\mathrm{a} 2-\mathrm{a} 3+\mathrm{f} 1-\mathrm{f} 2-\mathrm{f} 3+\mathrm{e} 2-\mathrm{c} 1 \\
& M_{S} 1=a 1-a 2-a 3+c 1 \\
& C_{S} 2=M_{S} 1-c 2+b 1-b 2-b 3 \\
& \mathrm{M}_{\mathrm{S}} 2=\mathrm{M}_{\mathrm{S}} 1-\mathrm{c} 2+\mathrm{b} 3 \\
& M_{S} 3=\mathrm{M}_{\mathrm{S}} 2+\mathrm{d} 2
\end{aligned}
$$

Thus, the Total Merging and Cross Conflict traffic intensities are respectively:

$M_{S}=M_{S} 1+M_{S} 2+M_{S} 3$

$C_{S}=C_{S} 1+C_{S} 2$

Northbound Flow (Dugbe/Adamasingba to UI/Sango direction)
$\mathrm{C}_{\mathrm{N}} 1=\mathrm{d} 1-\mathrm{d} 2-\mathrm{d} 3+\mathrm{b} 1-\mathrm{b} 2-\mathrm{b} 3+\mathrm{c} 2-\mathrm{e} 1(8)$
$M_{N} 1=d 1-d 2-d 3+e 1$
$\mathrm{C}_{\mathrm{N}} 2=\mathrm{M}_{\mathrm{N}} 1-\mathrm{e} 2+\mathrm{f} 1-\mathrm{f} 2-\mathrm{f} 3$

$\mathrm{M}_{\mathrm{N}} 2=\mathrm{M}_{\mathrm{N}} 1-\mathrm{e} 2+\mathrm{f} 3$

$M_{N} 3=\mathrm{M}_{\mathrm{N}} 2+\mathrm{a} 2$

Thus, the Total Merging and Cross Conflict traffic intensities are respectively:

$M_{N}=M_{N} 1+M_{N} 2+M_{N} 3$

$C_{S}=C_{N} 1+C_{N} 2$

All these quantities were recorded as counted and computed on the Computation Excel Spreadsheet designed for the purpose.

Throughput Times Runs: Apart from the traffic volume counts, the study requires the computation of the throughput times of vehicles from one end of the grade separated approach to the other. The floating vehicle approach was used. Using this method, a vehicle equipped with GPS was driven from one end of the flyover to the other in both directions during each period tallying with the periods identified above for traffic volume counts and recorded for as many rounds of travel possible for 15 minutes segments within each period. Calibrated and programmed GPS 
tool adapted for this purpose was used to record the times and corresponding time recordings were taken.

Collation Method: Data collected for each fifteenminute segment of the seven hours of data collection totaling 28 such segments. For the traffic volume counts, the sum of the tally marks for each fifteen minutes were obtained for all turning movements counted. These volumes were summed up for each conflict point they pass through and the conflict traffic intensities were taken in lieu of the actual conflict intensities since, these volumes are used as surrogates for the latter. As for the throughput times obtained, the averages were calculated for each fifteen minutesegment to represent the throughput time for the fifteen-minute segment.

Methods of Data Fitting and Modeling: Classical Linear Regression Analysis was used in this study. The method was aimed at obtaining linear regression relationships with the data obtained for the traffic conflict volume, a surrogate for conflict intensities, and the measured throughput times in the direction of the grade separation provided, for different periods of the day (morning, off- and evening peak periods). These regression models predict throughput times in relation to conflict intensities in two cases which are to be compared in order to assess the effectiveness or otherwise of the grade separation provided.

The first group of regression models captures conflict intensities at-grade as it currently exists having separated the traffic on the flyover.

The second group of regression models captures conflict intensities with the grade separated traffic added and loaded to the existing at-grade traffic as if the grade separation has not been provided.
The two groups of models were compared at corresponding periods in other to make decision on the efficiency and effectiveness of the flyover and the grade separation provided or otherwise.

The Classical Linear Regression Model: The Classical Linear Regression model is a linear relationship (or equation) relating a dependent variable (that is to be predicted) to a set of explanatory or predictor variables.

$\mathrm{Y}=\mathrm{A}_{0}+\mathrm{A}_{1} \mathrm{x}_{1}+\mathrm{A}_{2} \mathrm{x}_{2}+\mathrm{A}_{3} \mathrm{x}_{3}+\ldots+\mathrm{A}_{\mathrm{n}} \mathrm{x}_{\mathrm{n}}$

where $\mathrm{Y}=$ The dependent variable to be predicted $\mathrm{x}_{1}, \mathrm{x}_{2}, \ldots \mathrm{x}_{\mathrm{n}}=$ set of predictor variables of causal factors or explanatory variables which explain the prediction.

$A_{0}, A_{1}, A_{2} \ldots A_{n}=$ Regression coefficients which are to be determined for the variations among the predictor or explanatory variables to be determined for and particular stream of data of $\mathrm{Y}$ and those of $\mathrm{x}_{1}, \mathrm{x}_{2}, \ldots \mathrm{x}_{\mathrm{n}}$.

In this work therefore, the dependent variables are the measured throughput times across the intersection measured period by period alongside the traffic flow volume at-grade and on flyover. The predictor variables are the Traffic Conflict Volumes measured and used as surrogates for actual Traffic Conflict intensities. With reference to Figure 1, there are clearly eight conflict points ( $(3$ Mergers - M1, M2 and M3), (2 Crosses - C1, C2) and (3 Diverges - D1, D2 and D3)) involved along the direction being investigated. These translate to 8 explanatory variables and the generalized Linear Regression Model in each direction can be represented as:

$$
\begin{gathered}
\mathrm{T}=\mathrm{B}_{0}+\mathrm{B}_{1} \mathrm{M} 1+\mathrm{B}_{2} \mathrm{M} 2+\mathrm{B}_{3} \mathrm{M} 3+\mathrm{B}_{4} \mathrm{C} 1+\mathrm{B}_{5} \mathrm{C} 2+\mathrm{B}_{6} \mathrm{D} 1+\mathrm{B}_{7} \mathrm{D} 2+\mathrm{B}_{8} \mathrm{D} 3 \\
\text { Where, in this case, } \mathrm{T}=\text { Throughput time variable; } \\
\mathrm{B}_{\mathrm{i}}=\text { Regression coefficients for explantory variables } \mathrm{i}, \mathrm{i}=0,1,2 \ldots, 8
\end{gathered}
$$

However, diverging conflict points are conflict resolution points. They contribute little or nothing to delay and hence throughput time across the intersection. For the purpose of this analysis, the contributions of diverging conflict points are played down. Thus the resulting applicable model depends on the remaining five cross- and merging-conflict point traffic intensities, thus:

$\mathrm{T}=\mathrm{A}_{0}+\mathrm{A}_{1} \mathrm{M} 1+\mathrm{A}_{2} \mathrm{M} 2+\mathrm{A}_{3} \mathrm{M} 3+\mathrm{A}_{4} \mathrm{C} 1+\mathrm{A}_{5} \mathrm{C} 2 \quad 17$

There are then 6 regression coefficients, $A_{0}, A_{2}, \ldots$, $\mathrm{A}_{5}$ to account for.
The number of explanatory variables in the prediction model can further be reduced by computing crosscorrelation matrices for the variables involved as informed by the data in use. This was necessary to ascertain which of the variables explain the regression equation well, and to identify high co-linearity among the predictor variables. Thus, if two or more variables have very high cross-correlation explaining the independent variable, it suffices that just one of them is used for prediction in as much as the variable to be used is also highly correlated with the dependent variable. 


\section{RESULTS AND DISCUSSION}

Analysis of Current Traffic Conflict Flow Intensities: The intensities of traffic flow involved at the conflict points at-grade computed based on turning movements using (Equations $1-14$ ) and measured throughput times were analyzed with regression to predict throughput time. Cross-correlation analyses on the conflict intensity variables for both northbound and southbound traffic were performed to detect colinearity within them and with the dependent Throughput Time variable TT.

Existing Northbound Traffic Conflicts' Regression Analysis: For northbound traffic, it is evident the data streams for each of the conflict traffic intensity distributions over time are random with Coefficient of Variation (COV) greater than $20 \%$. In particular, the merger conflicts at-grade on this side of the traffic corridor are more intense with a Mean over 15 minuteperiods of 454.67 (3357.17 per hour) and wider variation with a Standard Deviation of 189 (919.08 hourly) vehicles compared to the Cross Conflicts' Mean of 335.08 (2366 per hour) and standard Deviation of 101.07 (306.32) vehicles. The implication of these is that merger conflicts pose the greatest threat to free flow of traffic on the at-grade intersection of the flyover complex. Remedial measures of treating merger conflicts will thus go a long way to improve the intersection performance atgrade.

The best combination of variables for existing conflict traffic flow predicting throughput time (TT) in the northbound corridor is $(\mathrm{CN} 1)$. The resulting equation with $\mathrm{R}^{2}$ of 0.64 is thus:

$\mathrm{TT}=0.931+0.010 \mathrm{CN} 1$

Existing Southbound Traffic Conflicts' Regression Analysis: The Southbound traffic corridor exhibits similar pattern of conflict traffic intensity as the northbound traffic corridor at-grade. The mean flows and coefficients of variation are however lesser indicating that conflict intensities at that corridor are less intense and also more predictable than on the northbound corridor. The conflict traffic intensity distributions over fifteen-minute time period are also random. The intensities of merger conflicts at-grade also predominate other types of conflicts at-grade. The best combination of variables for existing conflict traffic flow predicting throughput time (TT) in the southbound corridor is (MS2). The resulting equation with $\mathrm{R}^{2}$ of 0.495 is thus:

$\mathrm{TT}=0.802+0.005 \mathrm{MS} 2$

Analysis of Reloaded Traffic Conflict Flow Intensities: The flow intensities and throughput times at-grade were augmented with the flows on the flyover and estimated throughput times resulting from this to simulate a situation in which the flyover is not in place and all traffic flow at-grade.

Basic Assumptions for augmenting the At-Grade Flow: With regards to augmenting the at-grade corridor with flow on the flyover added with the existing flow, a few assumptions were made: (i) all traffic plying the flyover is adjudged as captive traffic which would have passed through the defunct intersection without the flyover in the directions they ply now. (ii) Traffic throughput times are proportional to intensities of flow (iii) The traffic flows at crossing directions to the intersection eastbound and westbound remain as they are currently as when the flyover was not in place (iv) Based on the rule of giving way to approaching traffic on the left at an uncontrolled junction, delays are only possible at 3 out of the 5 critical conflict points and the obvious fact that delays at 2 of them are merger conflicts and the other one a cross conflict point with postulated ratio of delays 1:2 for cross and merger conflicts respectively (v) Because of assumption 4 , for 5 conflict points comprising of 2 crosses and 3 mergers weights of delay intensities is 7 . For 2 mergers and 1 cross conflicts giving way to opposing traffic, the sum is 4 . Thus, the proportionate delay due to flyover traffic reloaded on the at-grade corridor, a factor of 4/7 was applied for reduction.

Derivation of the Augmented Throughput Times: The throughput times due to at-grade conflict flow intensities augmented with traffic on the flyover was obtained using the estimate of traffic that will actually go through the intersection from one end to the other. This was obtained for the northbound and southbound traffic turning movement counts (Figure 1) as in the equations below.

Throughfare Traffic $($ Northbound $)=(d 1-d 3)-(e 2-e 1)$

Throughfare Traffic (Southbound $)=(a 1-a 3)-(c 2-c 1)$

The measured at-grade flow was rated as throughput times per vehicle and this rate was used to multiply the additional traffic from the flyover and factored based on assumptions made to obtain increment of traffic throughput time for each direction and for each period. 
Augmented Northbound Traffic Conflicts' Regression Analysis: The best equation from cross-correlation analysis among regression variables of traffic northbound when the at-grade-intersection was augmented and reloaded with traffic on flyover is :

$\mathrm{TT}=0.896+0.008 \mathrm{CN} 1$

Augmented Southbound Traffic Conflicts' Regression Analysis: The best equation from cross-correlation analysis among regression variables of traffic southbound when the at-grade-intersection was augmented and reloaded with traffic on flyover is:

$\mathrm{TT}=0.971+0.004 \mathrm{MS} 2$

Predicted Throughput Times: Based on the derived regression equations (Equations 18, 19, 22 and 23), values of throughput times (in minutes) were computed for all cases and all periods as tabulated in Table below.

Table 1: Predicted Throughput Times At-Grade of the Intersection

\begin{tabular}{|c|c|c|c|c|c|}
\hline \multirow{2}{*}{\multicolumn{2}{|c|}{ Time Segments }} & \multicolumn{2}{|c|}{ Northbound } & \multicolumn{2}{|c|}{ Southbound } \\
\hline & & Current & Augmented & Current & Augmented \\
\hline \multirow{4}{*}{7 - 8 AM } & $7.00-7.15$ & 1.89 & 2.25 & 1.1 & 1.62 \\
\hline & $7.15-7.30$ & 2.25 & 2.56 & 1.2 & 1.64 \\
\hline & $7.30-7.45$ & 2.41 & 3.04 & 1.14 & 1.76 \\
\hline & $7.45-8.00$ & 2.19 & 2.71 & 1.14 & 1.74 \\
\hline \multirow{4}{*}{8 - 9 AM } & $9.00-9.15$ & 2.01 & 2.34 & 1.13 & 1.62 \\
\hline & $9.15-9.30$ & 2.21 & 2.63 & 1.17 & 1.71 \\
\hline & $9.30-9.45$ & 2.1 & 2.52 & 1.16 & 1.68 \\
\hline & $9.45-10.00$ & 2.37 & 2.77 & 1.16 & 1.69 \\
\hline & $12.00-12.15$ & 1.54 & 2.22 & 1.72 & 2.07 \\
\hline $12-1$ & $12.15-12.30$ & 1.89 & 2.52 & 1.47 & 2 \\
\hline & $12.30-12.45$ & 1.94 & 2.61 & 1.94 & 2.43 \\
\hline & $12.45-01.00$ & 2.25 & 2.88 & 2.21 & 2.71 \\
\hline & $02.00-02.15$ & 2.37 & 2.78 & 1.69 & 2.09 \\
\hline \multirow{3}{*}{1 - 2 PM } & $02.15-02.30$ & 1.97 & 2.58 & 1.22 & 1.73 \\
\hline & $02.30-02.45$ & 2.98 & 3.41 & 1.38 & 1.89 \\
\hline & $02.45-03.00$ & 1.61 & 2.44 & 2.01 & 2.37 \\
\hline \multirow{3}{*}{4 - 5 PM } & $04.00-04.15$ & 1.74 & 3.56 & 1.83 & 2.34 \\
\hline & $04.15-04.30$ & 2.75 & 3.51 & 1.74 & 2.24 \\
\hline & $04.30-04.45$ & 1.91 & 2.56 & 1.46 & 1.83 \\
\hline \multirow{8}{*}{5 - 6 PM } & $04.45-05.00$ & 3.2 & 3.81 & 2.02 & 2.53 \\
\hline & 06.00 - 06.15 & 1.17 & 2.09 & 2.31 & 2.68 \\
\hline & $06.15-06.30$ & 1.32 & 2.36 & 2.28 & 2.56 \\
\hline & $06.30-06.45$ & 1.2 & 2.4 & 2.19 & 2.58 \\
\hline & $06.45-07.00$ & 1.82 & 2.75 & 2.15 & 2.53 \\
\hline & Variance & 0.255 & 0.201 & 0.191 & 0.150 \\
\hline & Mean & 2.045 & 2.721 & 1.618 & 2.085 \\
\hline & COV & 12.461 & 7.387 & 11.807 & 7.201 \\
\hline
\end{tabular}

Normality test and test of difference for throughput times: Some comparative analysis tests were run on the predicted Current and Augmented (without flyover) throughput times which represents the current flow and a simulated scenario, as if the flyover is not in place. Normality tests were performed on the throughput times using Shapiro-Wilks test. The pvalue for the tests are as follows: current Northbound flow (p-value $=0.732)$, Augmented Northbound (without flyover) (p-value $=0.018)$, Current Southbound flow (p-value $=0.011)$, and Augmented Southbound flow (without flyover) (p-value $=0.016$ ) The results show that only northbound existing follows a normal distribution while the other three scenarios are non-normal. Consequently, a nonparametric test, Wilcoxon signed-rank test was adopted to determine whether throughput times for existing (Current) and augmented (without flyover) conditions were statistically different in the case of Northbound and Southbound directions. Wilcoxon signed rank test between existing and augmented throughput times for both northbound and southbound resulted in p-values of $<0.0001$ at alpha level of 0.05 . Additionally, throughput time difference analysis for northbound travel resulted in mean of 0.67 minutes $/ 40.2$ seconds and standard deviation of 0.07 minutes $/ 4.2$ seconds; a reduction by $33 \%$. Similarly, for Southbound travel, the mean was 0.46 minutes $/ 27.6$ seconds and standard deviation was 0.017 minutes $/ 1$ seconds; a reduction by $28.8 \%$. The results have shown that the difference between existing and augmented (without flyover) throughput time for both northbound and southbound were statistically significant. Thus, the flyover resulted in significant reduction in throughput times at Mokola intersection complex. 
Conclusion: The performance appraisal of the intersection complex presented in this work showed significant reductions in throughput times as a result of the construction of the flyover. Throughput times followed a non-normal distribution; hence care should be taken during simulation or predictive exercises. The current flow of traffic on the flyover is relatively low, suggesting significant marginal benefits are to be expected with additional traffic growth in the future. This work has provided a basic framework to assess the operational performance and benefits of the grade separated intersection in the event that a before and after study is too difficult or impossible to conduct.

\section{REFERENCES}

Alejandro, A; Mark, H (2003). A method for analyzing the performance of signalized intersection from airborne imagery

Davis, G; Xiong, H (2007). Access to destinations: travel time estimation on arterials

Day, CM; Brennan, TM; Hainen, AM; Remias, SM; Bullock, DM (2012). Roadway system assessment using Bluetooth-based automatic vehicle identification travel time data. Joint Transportation Research Program Affiliated Rep
Federal Highway Administration (FHWA), (2007) Traffic analysis toolbox volume VI: Definition, Interpretation and Calculation of Traffic Analysis Tools Measures of Effectiveness

Federal Highway Administration (FHWA), (2008) Traffic Signal Timing Manual

Manual on Uniform Traffic Control Devices (MUTCD), 2009

Remias, S; Hainen, A; Day, C (2013) Performance characterization of arterial traffic flow with probe vehicle data. Transportation Research Record: Journal of the Transportation Research Board; 2380(2): 10-21

Texas A\&M Transport Institute, 2018

Thompson MK; Kwon OH; Park MJ (2009) The Application of Axiomatic Design Theory and Conflict Techniques for the Design of Intersection: PART I. Proceedings of ICAD. The Fifth International Conference on Axiomatic Design Campus de Caparica - March 25-27 